\title{
PROYECTO TUNING EN CHILE: ANÁLISIS DE PROCESOS DE INTERNACIONALIZACIÓN DE LA EDUCACIÓN SUPERIOR
}

\section{Antecedentes}

\section{Tuning: un proyecto en expansión}

\section{1.a. Internacionalización de la educación superior en el contexto europeo}

En el contexto de la integración europea, que se consolida a partir de la Declaración de Bolonia, en mayo de 2001, tuvo lugar en Bruselas el lanzamiento del proyecto Tuning ${ }^{\mathrm{i}}$ con el apoyo de la Comisión Europea, en el marco del proyecto Sócrates en el que participan unos treinta países europeos ${ }^{\mathrm{ii}}$.

Tuning surge con la finalidad de contribuir significativamente a la creación y desarrollo de un espacio europeo de educación superior. Otra de las fuerzas impulsoras de dicho proyecto, es: "La búsqueda de una mayor calidad en las universidades europeas, una calidad buscada conjuntamente y focalizada en los programas que llevarán a la consecución de titulaciones, su diseño y componentes"iii.

i Significa "afinar", "sintonizar las estructuras educativas de Europa".

ii El proyecto Sócrates tiene como principal objetivo desarrollar una Europa del conocimiento y, así, dar respuesta a los grandes retos que plantea este nuevo siglo, tales como: promover la educación a lo largo de toda la vida, fomentar el acceso de todos a la educación, adquirir calificaciones y competencias reconocidas. De este modo, el proyecto propugna la cooperación europea en todos los ámbitos de la educación: la movilidad entre países europeos, la elaboración de proyectos comunes, la creación de redes europeas (difusión de las ideas y de las prácticas correctas) o la realización de estudios y de análisis comparativos. En la práctica, Sócrates ofrece a las personas becas para estudiar, enseñar, seguir cursos de formación en el extranjero; presta a los centros educativos ayuda para poner en marcha proyectos pedagógicos y para intercambiar experiencias; concede a las asociaciones y a las ONGs ayudas a fin de organizar actividades sobre temas educativos, entre otras tareas.

iii Tuning Educational Structures in Europe. Disponible en http://www.relint.deusto.es/ TUNINGProject/spanish/index.htm 
En su diseño trabajaron más de cien reconocidos expertos europeos, para llegar a puntos de comprensión y confluencia.

Con el propósito de incrementar la movilidad de estudiantes y profesionales, el proyecto se orienta a impulsar a escala europea un alto nivel de convergencia de la educación superior, desarrollar perfiles profesionales, determinar puntos de referencia para definir competencias genéricas y específicas de cada disciplina, facilitar la transparencia de estructuras educativas e impulsar la innovación a través de la comunicación de experiencias, y crear redes europeas y una estructura curricular modelo.

Para ello se definieron, en primer lugar, un círculo interno y un círculo externo. El interno está formado por alrededor de cien universidades que participan en la implementación y el desarrollo del programa en cinco áreas temáticas (Administración de Empresas, Educación, Geología, Historia y Matemáticas), y en la conformación de redes temáticas en Física y Química; el externo, por instituciones interesadas en el proyecto, pero que no participan tan activamente como las anteriores.

Al momento de analizar la documentación correspondiente al proyecto Tuning Europa, éste presentaba dos fases en su implementación. Los objetivos principales de la primera fase eran:

- Buscar puntos de referencia para dar lugar al dinamismo en un contexto de alta movilidad de profesionales entre países. La finalidad era transparentar los títulos en el sentido de especificar las competencias que los egresados estarían en condiciones de desempeñar.

- Desarrollar dos líneas de acción: una orientada a especificar competencias genéricas que identifiquen los elementos compartidos comunes a cualquier titulación, y otra a definir competencias específicas relacionadas a cada área temática o disciplina. 
En la segunda fase, el proyecto se concentró en dos nuevas líneas de acción. La primera se vinculó al sistema de transferencia y acumulación de créditos, como medio para promover la movilidad de estudiantes. Desde Tuning, se entiende que "...sin un sistema de créditos fiable y basado en las horas de trabajo del estudiante, que sea entendido de la misma manera por todos los interesados, no pueden alcanzarse los objetivos de un espacio europeo de educación superior".

La siguiente línea de acción se relaciona con la calidad y los enfoques de enseñanza y aprendizaje, evaluación y rendimiento. Aquí la preocupación se focaliza en generar un ambiente apropiado para la producción y transferencia de conocimientos y competencias genéricas y específicas en cada disciplina, que garantice nuevos tipos de aprendizaje.

Actualmente, más de cien instituciones representativas de los países de la Unión Europa han participado en la primera (20002002) y en la segunda fase del proyecto (2003-2004), coordinadas por las universidades de Deusto (España) y Groningen (Países Bajos). Su expansión se lleva a cabo no sólo en Europa: es un proyecto en implementación en universidades latinoamericanas, con posibles proyecciones hacia África y Asia.

\section{1.b. Proyecto Alfa para América Latina}

La idea de crear algo similar al proyecto Tuning en América Latina surgió en el marco de la IV reunión de seguimiento del ALCUE ${ }^{i v}$, realizada en Córdoba, Argentina, en octubre de 2002. Un grupo de universidades europeas y latinoamericanas presentó el proyecto a la Comisión Europea a fines de octubre de 2003, siendo aprobado por ésta como Proyecto Alfa, bajo la coordinación de la Universidad de Deusto, España.

El Proyecto Alfa Tuning América Latina, cuenta con la participación de instituciones de 18 países de la región, entre los

iv América Latina, el Caribe y Unión Europea. 
cuales se encuentra Chile. Busca -al igual que en Europa- "afinar" las estructuras educativas iniciando un debate cuya meta es identificar e intercambiar información, y mejorar la colaboración para el desarrollo de la calidad, efectividad y transparencia. La iniciativa convoca a 62 instituciones de educación superior, elegidas por los ministerios de educación en función de criterios como excelencia nacional, capacidad de diálogo con otras universidades y peso significativo en el sistema (tamaño de la institución, trayectoria, credibilidad y autoridad académica).

Los objetivos que orientan el proyecto son contribuir al desarrollo de titulaciones fácilmente comparables y comprensibles, impulsar la importancia de un nivel de convergencia en educación superior, desarrollar perfiles profesionales en términos de competencias específicas y profesionales, crear redes y desarrollar una estructura curricular modelo, promoviendo la integración latinoamericana de titulaciones.

Tuning América Latina contempla las siguientes cuatro líneas de acción: definición de competencias genéricas y específicas, elaboración de enfoques de enseñanza y aprendizaje, establecimiento de créditos académicos y preocupación por la calidad de los programas.

En el contexto de los procesos de renovación curricular impulsados por el programa Mecesup (Programa de Mejoramiento de la Calidad de la Educación Superior, Ministerio de Educación) es donde la integración de universidades chilenas a las actividades del Proyecto Tuning América Latina es visualizada desde el gobierno como una oportunidad para potenciar dicha renovación, producir sinergias y lograr resultados en un menor plazo.

Los fines planteados por el Mecesup para la integración de las universidades chilenas a Tuning América Latina coinciden con los que orientan al proyecto europeo y latinoamericano: "Contribuir al desarrollo de las titulaciones fácilmente comparables y 
comprensibles en una forma articulada en toda América Latina". Entre las principales proyecciones que el gobierno de Chile explicita al sumarse a este proyecto se encuentran la posibilidad de transitar hacia una economía basada en el conocimiento, disponer de capital humano avanzado suficiente y capaz de llevar adelante investigación e innovación competitiva a nivel mundial, y generar flujos rentables de conocimiento que puedan movilizarse con efectividad en el mundo.

Como resultado de las postulaciones efectuadas por las universidades para participar en estos procesos, el Centro Nacional Chile del Proyecto Tuning América Latina -creado en el marco del programa Mecesup- resolvió recomendar dicho proyecto a cinco universidades, durante una primera fase iniciada en el 2005, cada una de las cuales desarrolla una o más de las áreas propuestas. Se trata de las siguientes instituciones:

- Universidad de Chile (Matemáticas).

- Pontificia Universidad Católica de Chile (Historia).

- Pontificia Universidad Católica de Valparaíso (Educación).

- Universidad de Talca (Administración de Empresas).

- Universidad Metropolitana de Ciencias de la Educación (Historia).

Durante una segunda fase de implementación, se integraron ocho universidades más. A su vez, en el marco del proyecto Mecesup se ha resuelto apoyar redes similares nacionales todo Chile para difundir el proyecto Tuning y replicar sus resultados.

En este contexto, y para identificar competencias genéricas y específicas consensuadas entre los coordinadores del programa de cada universidad, se elaboró una encuesta con la lista de competencias de la experiencia europea, con el fin de determinar las prioritarias para cada institución. Esta encuesta recogió las opiniones de cuatro grupos clave: estudiantes, egresados, académicos y empleadores. Los resultados fueron discutidos y analizados por los coordinadores de cada universidad participante 
de Tuning en América Latina. A partir de las conclusiones, se agregaron tres nuevas competencias relevantes para el contexto latinoamericano.

La encuesta sobre competencias genéricas se realizó en Chile en mayo de 2005, y en noviembre del mismo año la de competencias específicas. Esta última consideró cinco áreas y se efectuó en 20 universidades.

Entre las coincidencias con los resultados de otros países de América Latina se encuentra el orden de prioridades establecido en relación con las competencias consideradas de mayor y menor importancia. Mientras esta investigación se encuentra en proceso, se desarrollan las encuestas de las ocho nuevas áreas incorporadas.

En el marco de la implementación del programa Mecesup, se espera un diagnóstico que sea de "gran utilidad no sólo para el reconocimiento y la integración latinoamericana de titulaciones, sino también para los procesos de renovación curricular que se están iniciando en Chile, especialmente en lo que tiene relación con la definición de perfiles profesionales, flexibilidad de mallas curriculares e incorporación de nuevas metodologías de enseñanzaaprendizaje". La definición de competencias constituye uno de los aspectos claves de la vinculación entre el proyecto Tuning y el programa Mecesup.

2. El impacto de la Declaración de Bolonia en la definición de políticas públicas en educación superior

Para orientar nuestras indagaciones en torno a los procesos de implementación del proyecto Tuning en universidades chilenas y avanzar hacia la comprensión de sus posibles implicancias y proyecciones para Chile y América Latina, tomamos como referente la Declaración de Bolonia, de junio de 1999.

Dicha declaración establece un conjunto de líneas de acción conducentes a la creación de un espacio europeo de educación 
superior hacia el año 2010, donde Europa, como bloque, pueda aumentar sus niveles de competitividad y responder a la competencia de instituciones extranjeras con sede en territorio europeo. Resulta relevante no sólo porque establece el marco en el que tiene lugar el proyecto Tuning, sino también un conjunto de transformaciones históricas que revolucionan el modo de concebir e implementar las políticas públicas en educación superior, en el contexto de la globalización de los mercados.

Tomando en cuenta el análisis efectuado por Bruce y Muga (2005), nos detendremos en algunas redefiniciones fundamentales que plantea la declaración europea, por constituir nuestro referente más importante a la hora de analizar los procesos de internacionalización de la educación terciaria en Chile, a propósito de la implementación del proyecto Tuning en algunas universidades del país:

- La Declaración de Bolonia amplía el número de actores en el diseño de las políticas en materia de educación superior. A partir de Bolonia, las políticas públicas de educación superior se definen en el marco de una red de voluntades políticas donde se articulan diversos niveles de decisión: uno supranacional asociado con las políticas provenientes de la Unión Europea; otro nacional, que corresponde a las políticas nacionales, y un tercero, que corresponde a las instituciones educativas. Su relativo éxito se explica por la articulación lograda entre los distintos actores, por el grado de flexibilidad del que los estados e instituciones universitarias han gozado y por el apoyo económico que ha prestado la Unión Europea.

Al ampliar el número de actores participantes en la definición de las políticas de educación superior, la declaración europea instala el tema de la internacionalización en el ámbito nacional como una dimensión que busca retroalimentar la política pública y, en el ámbito de las respectivas universidades, como una estrategia integral de desarrollo institucional que afecta, directa o indirectamente, todas sus funciones (docencia, investigación y extensión). 
Estas redefiniciones en la forma de diseñar las políticas públicas interpelan a los modelos tradicionales con los que han operado los Estados nacionales en esta materia, ya que exige reducir las aprensiones y reservas acerca de la intervención de un agente supranacional en las políticas de la educación superior que históricamente ha sido de su dominio exclusivo.

Por otra parte, estos replanteamientos de las políticas públicas de educación superior también exigen redefiniciones al paradigma clásico de universidad tradicional y casi inmutable, que no resulta congruente con la flexibilidad y apertura que demandan los escenarios sociales, científicos y económicos actuales y futuros.

\section{- La Declaración de Bolonia amplía la visión tradicional de} internacionalización de la educación superior.

Si bien el proceso de Bolonia acentúa el predominio de la lógica económica en los procesos de internacionalización (ante la necesidad de incrementar la competitividad de la educación superior europea frente a otros sistemas como el estadounidense), amplía también el alcance de estos procesos, pasando de una visión tradicional, asociada exclusivamente a la movilidad de estudiantes, hacia otra más estratégica que guarda relación con el desarrollo integrado de políticas nacionales e institucionales.

En efecto, aumentar los niveles de competitividad de la educación superior, en el contexto de la sociedad del conocimiento, involucra no sólo la definición de competencias generales en los graduados de las universidades para atraer el mayor número de alumnos y académicos extranjeros (que ha ido cobrando cada vez mayor valor económico), sino también crecientes niveles de especialización e inversión en investigación avanzada, en innovación tecnológica, en la creación de redes institucionales e internacionales, en el desarrollo y uso de tecnología de la comunicación e información en la educación, por nombrar sólo algunas. Desde esta perspectiva, se entiende que la internacionalización de la educación superior 
resulta una herramienta clave para alcanzar altos niveles de competitividad sólo en la medida en que:

- Constituya un rasgo distintivo de las políticas públicas orientadas a fomentar el desarrollo articulado de las diferentes dimensiones involucradas (curriculares, institucionales, políticas, internacionales, culturales, tecnológicas, entre otras).

- Los Estados nacionales continúen siendo los actores vitales en el diseño y ejecución de las políticas públicas en esta materia, en el marco de redes internacionales que potencien estos procesos.

- Se vea acompañada por reformas en los sistemas de educación superior que impacten en el modo tradicional de entender la misión y gestión de la universidad contemporánea.

Tomando como referente las profundas transformaciones que definen el escenario de implementación del proyecto Tuning Europa, y ante la integración de cinco universidades chilenas al proyecto Tuning América Latina, surgen las preguntas centrales que orientan esta investigación:

- ¿Cuál es el impacto que la implementación del proyecto Tuning tiene sobre las dinámicas de desarrollo institucional en las universidades estudiadas? ¿Cuáles son las expectativas, riesgos y oportunidades que se vislumbran?

- ¿Cuál es el marco de las políticas públicas que regulan estos procesos?

- ¿Es posible pensar en Tuning América Latina como un espacio de articulación hacia la definición de políticas sistemáticas de educación superior?

\section{Metodología}

Para llevar a cabo esta investigación, se efectuó el análisis de la documentación vinculada al proyecto Tuning Europa, América Latina y Chile, a partir de la elaboración de una pauta referida a los procesos de internacionalización de la educación superior en Chile y en el extranjero. 
Se realizaron entrevistas en profundidad al coordinador del Centro Tuning en Chile y a cada actor responsable de la implementación del proyecto Tuning en sus respectivas universidades. Se eligieron las primeras cinco universidades del país que se encontraban implementando Tuning en alguna de sus carreras. Luego de seis entrevistas se logró recoger información que la revisión de literatura por sí sola no permitía documentar y, de esta forma, pudimos conocer algunas particularidades y dinámicas propias de la puesta en marcha del proyecto que eran significativas para la investigación.

Posteriormente, se analizaron dichas entrevistas, contrastando la información obtenida con los documentos revisados. La finalidad del cruce de información fue dilucidar aspectos relevantes y particulares que se presentan en el contexto de la implementación del proyecto Tuning en universidades del país, así como las tensiones y desafíos que un proyecto de esta envergadura involucra.

Esta investigación se inicia en octubre del 2005, cuando las universidades se encontraban en su segundo año de implementación del proyecto Tuning, y estaban por iniciar la segunda encuesta sobre competencias específicas. En diciembre del mismo año se llevaron a cabo las entrevistas en profundidad y durante 2006 se han realizado los análisis y la triangulación de información que dan lugar a las conclusiones de este trabajo.

\section{Resultados de la investigación}

4.1. El impacto de Proyecto Tuning en las universidades chilenas desde la perspectiva de los actores involucrados

Al indagar el modo en que se ha estado desarrollando la implementación del proyecto Tuning en las cinco primeras universidades chilenas que han iniciado estos procesos, se evidencian aspectos y dinámicas comunes, así como también matices y diferencias que en el marco de este trabajo nos interesa destacar: 
- En las cinco universidades queda explícito que el proyecto complementa un proceso de rediseño curricular emprendido previamente por las instituciones. No obstante, dicho proceso presenta en cada institución diferentes alcances en términos de magnitud y desarrollo. Al momento de iniciado el estudio, dos de estas universidades cumplían dos años de trabajo de reforma curricular en todas las carreras de pregrado, liderando estas iniciativas de reforma en el país; mientras que las tres universidades restantes se encontraban en el primer año de implementación de sus reformas curriculares, presentando menor grado de avance en esta materia.

- Si bien la integración de la universidad al proyecto se focaliza en una carrera concreta, sólo en dos casos la iniciativa de sumarse surge desde la carrera o facultad involucrada. En las tres universidades restantes, la iniciativa surge desde rectoría, como política institucional que asume la definición de un currículo por competencias para toda la universidad.

Al averiguar sobre la dinámica de conformación de los equipos responsables de la coordinación del proyecto en cada universidad, se ponen de manifiesto algunas particularidades:

- En ningún caso existen equipos encargados en forma exclusiva de la coordinación al interior de la universidad. Por lo general, el número de responsables no superan las dos personas, quienes, además, ocupan otros cargos y cumplen otras funciones en la institución.

- En tres de los casos estudiados las personas responsables pertenecen a la estructura organizacional de la facultad correspondiente, dependiendo, por lo general, del decanato o de la secretaría académica de la facultad. En los dos casos restantes, dependen de rectoría o, más específicamente, de las unidades a cargo de la reforma curricular impulsada por la división de asuntos académicos de la universidad.

Entre las tareas fundamentales que asumen estos equipos de trabajo destaca la difusión del proyecto al interior de las 
universidades. Respecto de ello, los actores reconocen que, por tratarse de un proyecto incipiente, la gran parte del cuerpo académico aún lo desconoce por completo. No obstante, es posible advertir diferentes grados de avance en este sentido, ya que la difusión y el desarrollo de la implementación del proyecto en cada universidad se encuentran estrechamente relacionados con el "respaldo institucional" del que disponen los equipos a cargo. Las universidades donde los coordinadores pertenecen a las unidades encargadas de la reforma curricular impulsada para toda la universidad presentan un notable avance respecto de la difusión al interior de los claustros académicos, en relación con aquellas donde la coordinación parte de una facultad o carrera concreta.

Acerca de las características y mecanismos de difusión utilizados en las distintas comunidades universitarias, también es posible encontrar matices. En tres universidades la difusión consiste predominantemente en seminarios, exposiciones, presentaciones, orientadas a lo que los entrevistados suelen llamar "la bajada" de la información, expresión que da cuenta de cierta unidireccionalidad en la relación con los cuerpos académicos. Por el contrario, en otra de las universidades abocadas al rediseño curricular en todas sus carreras de pregrado la información no sólo se "comunica", sino que también se "discute" a través de diversas comisiones de trabajo formadas por académicos, donde se definen las competencias específicas que se incorporarán en las mallas curriculares. Finalmente, en la última universidad los actores explicitan el hecho de no contar con mecanismos de difusión, por tratarse de procesos de reforma emprendidos recientemente.

Respecto del alcance de los cambios posibles ante la implementación del proyecto en sus respectivas instituciones, en todos los casos los actores visualizan su incidencia circunscrita exclusivamente a la definición de competencias genéricas y específicas. Llama la atención que otros aspectos contemplados dentro de las líneas de acción del proyecto -tales como el cambio de los enfoques y prácticas de enseñanza-aprendizaje vigentes- no 
sean percibidos por los entrevistados como ámbitos susceptibles de cambio en el marco de estos procesos de reforma.

A la hora de hablar de las principales motivaciones de las universidades para sumarse a estos procesos, los actores explicitan motivaciones de diversa índole. Entre ellas se destaca la búsqueda, por parte de las universidades, de recursos financieros en tiempos en que los fondos públicos no abundan. Desde esta perspectiva, unirse al proyecto Tuning América Latina no sólo ha significado para estas instituciones recibir importantes incentivos económicos, sino que, además, ahorro de dinero y trabajo.

En un segundo orden de prioridades se manifiestan motivaciones académicas, ya que el proyecto es percibido como un mecanismo que permite y facilita emprender transformaciones que resultan ineludibles para las universidades contemporáneas ante las demandas que plantea la sociedad del conocimiento. En este escenario, la posibilidad de crear redes de intercambio académico y científico, y mejorar la educación a través de la incorporación de estándares internacionales de calidad se encuentran entre las principales expectativas.

Finalmente, resultan recurrentes las motivaciones de índole "política". Los actores perciben como una ventaja que la definición de competencias genéricas y específicas se dé en un espacio europeo o latinoamericano, ya que, de esta manera, las instituciones "se evitan" tener que generar espacios de discusión y negociación con la comunidad universitaria. Así, desde la perspectiva de los entrevistados, la integración de las universidades les permite avanzar "más rápido" en la puesta en marcha de sus reformas curriculares, ya que dicho proyecto instala ante la academia perfiles y competencias profesionales ya definidos.

El último punto se tensiona con los problemas que los mismos entrevistados vislumbran en estos procesos, entre los que se destacan el temor de que la reforma "quede en el papel", sin que 
se lleguen a transformar las concepciones y prácticas instaladas históricamente en la vida universitaria.

Expresiones como las citadas resultan muy recurrentes en las entrevistas a la hora de hablar de los riesgos que se perciben de cara a la implementación de los cambios propuestos. La falta de definiciones claras respecto al "cómo" -esto es, metodologías y mecanismos concretos de implementación de la reforma-, la resistencia al cambio por parte de los claustros académicos, así como la verticalidad con la que se han tomado las decisiones en algunas instituciones, son los principales obstáculos que los entrevistados de las diferentes universidades coinciden en destacar.

4.2. Las percepciones de los actores universitarios respecto de la función de las políticas públicas en los procesos de internacionalización de la educación superior en Chile Con el fin de conocer el modo como opera el marco regulador de los procesos de internacionalización de la educación superior en Chile, indagamos la percepción de los actores institucionales en torno al papel que ha cumplido el Estado en el contexto de la integración de algunas universidades al proyecto, así como sus opiniones respecto a la definición de las políticas públicas en esta materia.

Los entrevistados coinciden en manifestar una valoración positiva respecto del rol del Mecesup ante la implementación del proyecto en las respectivas universidades. Entre los aspectos más valorados se encuentra la voluntad política por parte del Estado de apoyar estos procesos, manifiesta en el incremento de la asignación de recursos para fomentar procesos de renovación curricular y académica.

Lejos de sentir amenazada la autonomía institucional, las universidades que participan del proyecto destacan el respaldo institucional que esta presencia estatal supone. El hecho de que su implementación tenga lugar en el marco de políticas de gobierno, 
es percibido por los actores como una situación que pone a Chile en "un lugar privilegiado" respecto del resto de los países de América Latina.

\subsection{Tuning en Chile y su (des)articulación con América Latina}

Para conocer en qué medida Tuning América Latina es percibido como posible espacio de articulación hacia la definición de políticas conjuntas de educación superior, analizamos los documentos ministeriales e indagamos la opinión de los actores en relación con el tema.

En primer lugar, en cuanto a la integración de las universidades chilenas al proyecto, tanto en los documentos del programa Mecesup como en las entrevistas se pone de manifiesto una valoración positiva. Entre las ventajas percibidas se destaca la posibilidad de generar redes que permitan recibir información e intercambiar experiencias, motivar y formar especialistas nacionales, guiar las metodologías de trabajo y fomentar vínculos de colaboración y evaluación comparativa, entre otras.

No obstante, Tuning es valorado, en este contexto, sólo como insumo, como un referente más que aporta a los procesos de modernización curricular iniciados previamente en el país. Los actores aluden a la presencia de cierto liderazgo, por parte de Chile, en los procesos de internacionalización de la educación superior, respecto del resto de los países de América Latina.

Al indagar la opinión de los actores respecto de las posibilidades latinoamericanas de constituir un "espacio común de educación superior", éstos coinciden en manifestar sus dudas a la hora de pensar en los países de la región como comunidad. Mientras que los documentos del Ministerio de Educación aluden a la ausencia de "un marco político, como el de Bolonia en Europa, que defina y oriente este proceso", los entrevistados identifican serios obstáculos para la creación, a mediano plazo, de un espacio latinoamericano de articulación de educación superior, entre los cuales destacan el peso 
de ciertas "asimetrías culturales" entre los países, así como los diferentes niveles de desarrollo económico e institucional. Chile es percibido como un país que ocupa un lugar privilegiado por su grado de desarrollo y organización respecto al resto de América Latina.

También se pone de manifiesto el deseo de lograr, a mediano plazo, la articulación con universidades chilenas en el marco de un sistema de crédito transferible, orientado a lograr movilidad interna, tanto estudiantil como profesional. Sin embargo, esta vinculación se ve poco probable. Las razones se sustentan en la profunda segmentación del sistema de educación superior que, al evidenciar la coexistencia de diferentes niveles de calidad en la oferta, establece un sistema de jerarquías entre universidades, dificultando las proyecciones hacia la implementación de un futuro sistema de transferencia en el ámbito nacional.

\section{Discusión de los resultados}

Tomando como referente los procesos de internacionalización de la educación superior en los países europeos, en el marco de la implementación del proyecto Tuning Europa, es posible notar que, en Chile, si bien hay avances importantes en este campo, aún son importantes los desafíos que se presentan para alcanzar los estándares de los países desarrollados y las tareas pendientes son considerables.

En materia de internacionalización de la educación superior, entre los aspectos que constituyen una ventaja importante para el país en relación con los países latinoamericanos, se destaca la voluntad política por parte del Estado de promover procesos sistemáticos de internacionalización de la educación superior en sus tres niveles: estatal, institucional e internacional. Esta presencia estatal se manifiesta en:

- El diseño e implementación de una política pública de reforma sistemática de la educación terciaria que, con vistas a 
incrementar la efectividad y calidad del sistema, plantea la internacionalización de la educación superior como uno de sus ejes fundamentales.

- El incremento de la asignación de recursos, para fomentar, a partir de un fondo competitivo, procesos de renovación académica y curricular en las universidades del país, como estrategia orientada tanto a la sintonización y modernización de la oferta de educación superior, como a favorecer la mejora de los procesos de gestión institucional.

- El reconocimiento explícito por parte del gobierno chileno hacia el proyecto Tuning América Latina, como oportunidad para producir sinergias, fomentar la formación de redes de colaboración, permitir evaluaciones comparativas y potenciar los procesos de renovación curricular impulsados por el programa Mecesup en universidades chilenas.

En este sentido, las políticas emprendidas por el gobierno de Chile en materia de internacionalización de la educación superior asumen la visión que se instala a partir de la Declaración de Bolonia respecto del rol protagónico del Estado en estos procesos, tanto como ente articulador con instancias internacionales, como en su papel promotor de estrategias integrales de desarrollo para las universidades del país.

Entre otras de las ventajas se encuentra el hecho de que las primeras universidades chilenas que se integraron al proyecto Tuning América Latina ya habían iniciado procesos de renovación curricular en la línea de formación por competencias, lo cual demuestra cierta interiorización por su parte respecto de las demandas que plantea la sociedad del conocimiento a la educación superior. No obstante, cabe aclarar que los grados de avance en esta materia varían entre las universidades estudiadas, planteándose mayor apropiación de la reforma curricular en aquellas en las que el rediseño ha sido planteado por sus autoridades máximas como "política" o "sello" para toda la universidad. 
Por otra parte, nos interesa detenernos en el análisis de dos problemas fundamentales: el concepto de internacionalización que opera en las prácticas institucionales y las particularidades que presenta el proceso de implementación de Tuning en América Latina.

En relación con el primero, en las instituciones de educación superior estudiadas, prevalece una concepción restringida de los procesos de internacionalización de educación superior. Al explorar las dinámicas institucionales en el marco de estos procesos se evidencia que, en la mayoría de los casos, Tuning es un proyecto aún desconocido por gran parte de las comunidades universitarias involucradas, lo cual reduce su capacidad transformadora sobre las prácticas tradicionales instaladas en la institución.

En aquellas universidades en que se implementaron actividades de difusión del proyecto, predominan metodologías "verticalistas" de trabajo, centradas en la difusión de la información, sin contemplar la generación de espacios para la discusión y, en definitiva, la apropiación por parte del cuerpo académico de la envergadura de los cambios esperados.

Lo anterior queda de manifiesto cuando los entrevistados hacen referencia al impacto del proyecto Tuning en sus respectivas instituciones. Los actores sólo visualizan su incidencia circunscrita exclusivamente a la definición de competencias genéricas y específicas, sin considerar y dimensionar su impacto como proyecto orientado hacia una reforma curricular. Se suma a ello, la omisión en las declaraciones de aspectos fundamentales relacionados con la incorporación de elementos culturales inherentes a este proceso.

Tomando en cuenta los aspectos antes mencionados, pareciera prevalecer en las políticas institucionales emprendidas una visión "restringida" de los procesos de internacionalización de la educación superior, ya que el cambio se limita a la definición de competencias profesionales, sin afectar las dinámicas culturales, 
organizativas y de gestión que resulta necesario revisar para garantizar el logro de las transformaciones emprendidas.

Desde perspectivas actuales, internacionalizar la oferta educativa es mucho más que asumir perfiles y competencias profesionales definidas según estándares internacionales. Supone no sólo una política universitaria que oriente una reforma curricular con dimensión internacional, sino también una estrategia de incorporación de toda la comunidad académica y un sentido compartido que impacte en los pilares de la labor universitaria (investigación, docencia, extensión).

El problema es que, sin un conjunto definido de estrategias integrales de desarrollo institucional, orientadas a conectar las actividades aisladas, los procesos de internacionalización de la formación tienden a ser marginales, favoreciendo áreas específicas y grupos particulares de alumnos y de facultades.

El riesgo que se advierte es que, mientras estos temas son analizados y debatidos en las instituciones de países desarrollados, en las nuestras el tema está lejos de visualizarse como tal, lo que aumenta la desigualdad de oportunidades entre quienes tienen acceso a formarse con una amplia visión internacional y quienes no lo tienen.

Respecto de las particularidades de la implementación del proyecto Tuning en América Latina, la ausencia de políticas marco que orienten las acciones hacia un sistema de educación superior articulado establece condiciones desfavorables en relación con los países europeos.

En efecto, al contar con Bolonia como telón de fondo, el proyecto europeo tiene un marco más preciso, basado en una red de voluntades políticas. Respecto del tema, los documentos latinoamericanos reconocen que América Latina no tiene fecha para determinar un espacio de educación superior, tampoco cuenta con 
un marco político tan encaminado como el que posee Europa. A pesar de ello, las expectativas de "crear puentes y lenguajes comunes" ya son parte del proceso de implementación del programa Tuning. Mientras que en Europa se otorga especial importancia al Sistema Europeo de Transferencia de Créditos (ECTS), en el caso latinoamericano las expectativas se centran sólo en la búsqueda de puntos comunes de referencia, poniendo énfasis en la definición de competencias generales y específicas, con la finalidad de facilitar la movilidad de los estudiantes y profesionales universitarios.

De este modo, mientras Bolonia amplía el concepto de internacionalización invitando a analizar esta modalidad en términos de cooperación (sello político) para aumentar niveles de competitividad, (sello económico), América Latina carece de un espacio articulado que aumente su capacidad competitiva, ya que Tuning en la región sólo constituye una iniciativa de instituciones, sin contar con el respaldo de acuerdo políticos internacionales. De no modificarse esta tendencia, los países de Latinoamérica tendrán que seguir enfrentando problemas tales como fuga de cerebros, aumento de la segmentación del sistema y desigualdad de oportunidades, profundizando el riesgo de convertir la implementación de este tipo de programas en una nueva forma de colonización.

\section{Conclusión}

La implementación del proyecto Tuning en nuestro país se presenta como un caso incipiente de internacionalización de la educación superior en Chile, proceso que posee características complejas y de impacto entre los países de la región, y del cual las universidades no pueden estar ajenas.

A partir de nuestras indagaciones, consideramos relevante situar algunos aspectos que demandan ser revisados en un marco de discusión y debate para el futuro desarrollo de los procesos emprendidos, tales como, las dinámicas instaladas en las 
universidades contemporáneas, el rol de las políticas públicas y la conceptualización de internacionalización en el marco de la educación superior.

Con relación al primer punto, sin bien las universidades donde se realizó el estudio se encuentran en una etapa inicial de trabajo, creemos importante reflexionar en torno a los siguientes temas:

- La necesidad de repensar una reforma curricular participativa, que garantice una internalización real de los procesos de cambio por parte de los actores involucrados.

- Tuning, como la presentación de un proyecto de reforma curricular que intenta definir titulaciones comparables, exige que la preocupación por los procesos de internacionalización en las universidades transite de ser un tema marginal a un tema transversal, que impacte sus tres pilares fundamentales: docencia, investigación y extensión.

En relación con el rol de las políticas públicas, el Ministerio de Educación ha mostrado voluntad política al promover la renovación curricular en las instituciones, mediante una fuerte asignación de recursos. No obstante, quedan tareas pendientes. Por una parte, es necesario continuar y enfatizar los procesos de aseguramiento de la calidad, evitando de este modo el aumento de la segmentación del sistema. Por otra parte, parece impostergable involucrar a más actores que representen los diferentes niveles del quehacer universitario en la definición de estos procesos, y generar espacios de discusión que se orienten a desarrollar una estrategia integral que articule los distintos niveles de decisión. Sin lugar a dudas, la envergadura de las transformaciones emprendidas, que impactan en los modos tradicionales de entender la misión de la universidad contemporánea, exige un debate público respecto del rumbo que tome la implementación de este proyecto.

En cuanto a la conceptualización de internacionalización de la educación superior, Tuning en Chile aún mantiene una visión 
tradicional centrada en la definición de competencias, de acuerdo a los estándares requeridos por la empresa y la movilidad estudiantil. Es indiscutible que la internacionalización involucra mucho más que lo mencionado y resulta clave ampliar la mirada hacia una visión estratégica que guarde relación con las políticas institucionales y nacionales, la creación de redes, el desarrollo de investigación avanzada, y el uso de tecnologías de la comunicación, entre otros. Para ello las alianzas con respaldo político resultan fundamentales.

Finalmente, y a propósito de una mirada de internacionalización de la educación superior más amplia y acorde con los desafíos presentes y futuros, es necesario desarrollar políticas culturales, nacionales e institucionales, orientadas hacia las diversidades presentes en las universidades del país y de escaso reconocimiento por parte de sus autoridades. En efecto, los procesos de internacionalización de la educación superior nos ponen de cara a un modelo de universidad que se abre al mundo y que ya no responde exclusivamente a las necesidades de su contexto inmediato.

\section{Bibliografía}

Bruce, G. y Muga, A. (2005) La declaración de Bolonia y su impacto para las políticas públicas en educación superior: internacionalización y competitividad en un mundo globalizante. Calidad en la Educación, Chile, 22, pp. 259-175.

Casas Armengol, M. (2005) Nueva universidad ante la sociedad del conocimiento. Revista de Universidad y Sociedad del Conocimiento, 2 (2). Disponible en http://www.uoc.edu/rusc/2/2/dt/esp/casas.pdf

González, L. (2003) Los nuevos proveedores externos de educación superior en Chile. Santiago de Chile: Instituto de Educación Superior para América Latina y el Caribe.

González, J. y Wagenaar, R. (2005) Tuning-América Latina: un proyecto de las universidades. Revista Iberoamericana de Educación, 35, pp. 151164.

Knight, J. (2002) Trade in Higher Education Services the Implications of Gats. Toronto: OISE. 
Knight, J. (2003) Updating the definition of internationalization. International Higher Education. Disponible en http://www.bc.edu/ bc_org/avp/soe/cihe/newsletter/news33/text001.htm

Poblete, A. y Zúñiga, M. (2002) Internacionalización de la educación superior: alcances culturales para la cooperación. Santiago de Chile: Cinda, pp. 227-250.

Rizvi, F. (2000) Postcolonial perspective on globalization and education. Illinois: University of Illinois at Urbana-Champaign.

Recibido: 30 de mayo de 2006

Aceptado: 14 de junio de 2006 